\title{
Subsystem purity as an enforcer of entanglement
}

\author{
S. Bose,$^{\dagger}$ I. Fuentes-Guridi, ${ }^{*}$ P.L.Knight, ${ }^{*}$ and V. Vedral* \\ * Optics Section, The Blackett Laboratory, Imperial College, London SW7 $2 B W$, United Kingdom \\ ${ }^{\dagger}$ Centre for Quantum Computation, Clarendon Laboratory, University of Oxford, Parks Road, Oxford OX1 3PU, England
}

\begin{abstract}
We show that entanglement can always arise in the interaction of an arbitrarily large system in any mixed state with a single qubit in a pure state. This small initial purity is enough to enforce entanglement even when the total entropy is close to maximum. We demonstrate this feature using the Jaynes-Cummings interaction of a two level atom in a pure state with a field in a thermal state at an arbitrarily high temperature. We find the time and temperature variation of a lower bound on the amount of entanglement produced and study the classical correlations quantified by the mutual information.
\end{abstract}

Entanglement is a key resource in quantum information processing [1,2]. In practice, due to decoherence, it is very difficult to generate and maintain entanglement in the form of a pure state. With this motivation, there has been a lot of recent interest in understanding and quantifying entanglement of mixed states 3 . 5. As entanglement is an important resource, we need to investigate whether and how it can be generated in severe conditions of mixedness [3]. Interestingly, if we had a qubit in a completely pure state $|0\rangle_{1}$ and another qubit in a completely mixed state $(1 / 2)(|0\rangle\langle 0|+| 1\rangle\langle 1|)_{2}$, we could let them interact through

$$
\begin{aligned}
& |0\rangle_{1}|0\rangle_{2} \rightarrow|0\rangle_{1}|0\rangle_{2} \\
& |0\rangle_{1}|1\rangle_{2} \rightarrow\left|\psi^{+}\right\rangle_{12},
\end{aligned}
$$

where $\left|\psi^{+}\right\rangle_{12}=\frac{1}{2}\left(|01\rangle_{12}+|10\rangle_{12}\right)$, to generate an entangled state. Surprisingly, this also holds for a pure qubit interacting with a fully mixed state of an arbitrary dimensional (even macroscopic) system. In this letter, we demonstrate this in the context of a pure state atom interacting with mixed state quantum field (an infinite dimensional system) through the Jaynes-Cummings model.

The Jaynes Cummings model (JCM) [6] if is the simplest model that describes the interaction between light and matter. It consists of a two level atom interacting with a near-resonant quantized mode of the field. The atom and field become dynamically entangled by their interaction. This model provides direct evidence for the quantum mechanical nature of the electromagnetic field by predicting collapses and revivals of Rabi oscillations of the atom [8]. These have been tested experimentally [9,10]. The JCM has analytical solutions for arbitrary coupling constants and can easily be extended to include a variety of initial conditions [11], dissipation and damping [12], multiple atoms [13], multilevel atoms [14], multimode fields [15] and more elaborate interactions [16]. For certain pure initial states of the atom and the cavity mode, the JCM produces entanglement which oscillates with time [17]. In this paper we show that even if the cavity field is initially in a thermal state, entanglement still arises in course of the JCM interaction. The result holds irrespective of the temperature of the field. This goes counter to the folklore that entanglement, being a very quantum attribute, should automatically disappear at high temperatures. We give a lower bound on the entanglement produced as a function of the temperature of the cavity field and time. We also compare the entanglement, which quantifies the quantum part of the correlations, to the total correlations as quantified by the mutual information.

Entanglement is well understood for pure states of bipartite systems such as the JCM. The general state of a system involving two subsystems (say atom and field) can be written as a superposition of the products of individual states. If the state is pure it can always be written in the Schmidt form 18]

$$
|\Psi\rangle=\sum_{n} g_{n}\left|u_{n}^{\prime}\right\rangle_{a}\left|v_{n}^{\prime}\right\rangle_{f}
$$

where $\left|u_{n}^{\prime}\right\rangle_{a}$ and $\left|u_{n}^{\prime}\right\rangle_{f}$ are orthonormal bases for the atomic and field subsystems respectively. The correlations of the two systems are then fully displayed. In this case (pure bipartite states), entanglement is quantified by the entropy of the reduced density matrix of either of the subsystems defined as $S(\rho)=-\operatorname{Tr}\left(\rho_{a} \log \left(\rho_{a}\right)\right)$. This quantification of entanglement has been used in all earlier studies of entanglement in JCM with pure initial states [17]. For the JCM with both pure atomic and cavity field states, the cavity field can be considered as an effective two level system [17].

Here we are interested in studying entanglement for an initial mixed state of the cavity field, as mixed states are the true representation of the state of the field at a finite temperature. Entanglement for mixed states is difficult to define. This is because we cannot easily define an analogue of the Schmidt decomposition for a general mixed state of a composite system. Such a mixed state can be expanded in terms of pure states in infinitely many different ways and it is not clear which, if any decomposition should be favored. Mixing two entangled pure 
states could result in a mixed state with entanglement much less than the average entanglement of the states mixed. Mixed state entanglement is thus a very different entity to either correlations or pure state entanglement. At least three different measures have been used to quantify entanglement for a mixed state. One of these measures, the relative entropy of entanglement [3], is defined for mixed state $\rho_{a f}$ of a composite system (such as the atom-field system in the JCM) as

$$
E_{r e}\left(\rho_{a f}\right)=\min _{\sigma \in D}\left(\operatorname{Tr}\left(\rho_{a f}\left(\log \rho_{a f}-\log \sigma_{a f}\right)\right)\right),
$$

where $D$ is the set of disentangled (separable) states of the system. A disentangled state can be written in the form $\sum_{i} p_{i} \rho_{a}^{i} \otimes \rho_{f}^{i}$. This measure tells us how difficult it is to distinguish the given entangled state from its closest approximation to the set of separable states. The other measures of entanglement are associated with formation and distillation of entangled states. Consider the number, $n$, of copies of a non maximally entangled state $\rho_{a f}$ that can be created by using only correlated local actions (i.e. through correlated actions on the field state and atomic state alone) on a number, m, of maximally entangled states. Entanglement of formation is the asymptotic conversion ratio, $m / n$ in the limit of infinitely many copies 19,

$$
E_{f}\left(\rho_{a f}\right)=\min \sum_{i} p_{i} S\left(\rho_{a}^{i}\right)
$$

where the minimum is taken over all the possible realizations of the state $\rho_{a f}=\sum_{i} p_{i}\left|\Psi_{a f}^{i}\right\rangle\left\langle\Psi_{a f}^{i}\right|$. A measure stemming from the opposite process (distillation) is the entanglement of distillation. It is the asymptotic rate $m / n$ of converting $m$ copies of a non-maximally entangled state $\rho_{a f}$ into $n$ copies of a maximally entangled state by means of correlated local actions. The entanglement of distillation is in general smaller than formation. All the different measures of entanglement are related to each other through the amount of available classical information about the decomposition of the state [20].

It is not easy to compute the value of entanglement from the measures. The entanglement of formation is the only measure for which an analytical method exists for calculating the entanglement, but this is specific to the case a state of $2 \times 2$ systems. However, in our case there is a two level atom interacting with a cavity field, which is an infinite dimensional system. For such states, we can still give a lower bound on the entanglement from the known result in the case of $2 \times 2$ systems. We first project the entire atom-field state onto a subspace equivalent to a $2 \times 2$ system. We can then compute the entanglement of formation for each of the outcomes. This particular projection onto a $2 \times 2$ system, as we will show, can be done by local actions alone. Being local, such an action cannot increase the entanglement on average [3]. If we compute the average of the entanglement over all possible outcomes, the result will thus be a lower bound on the entanglement in the initial $2 \times \infty$ state of the atom and the cavity field. We will also look at the total correlations between the atom and the mixed field as quantified by the mutual information [21],

$$
I=S\left(\rho_{a}\right)+S\left(\rho_{f}\right)-S\left(\rho_{a f}\right)
$$

where $\rho_{a}$ and $\rho_{f}$ are the reduced density matrices of the atom and field. This measure quantifies how much the correlated systems know about the state of each other. As the mutual information indicates the total correlations, it should be larger than the lower bound on entanglement we compute. We will compare the two quantities (i.e. the lower bound on the entanglement and the mutual information) to understand how much the purely quantum correlations contribute to the total correlations.

We consider the field in our JCM example initially to be in a thermal state at some temperature $T$ with the probability distribution $P_{n}$ for number states $|n\rangle$ being given by

$$
P_{n}=\frac{1}{1+\langle n\rangle}\left(\frac{\langle n\rangle}{1+\langle n\rangle}\right)^{n}
$$

where $\langle n\rangle=\left\{e^{\beta \hbar \omega}-1\right\}^{-1}$ is the mean photon number, $\beta=1 / k_{B} T$ and $k_{B}$ is Boltzmann's constant, $T$ is the temperature, $\omega$ the frequency of the optical mode and $\hbar$ the Planck's constant. The two-level atom is initially taken to be in the excited state $|e\rangle_{a}$ (the ground state being $|g\rangle_{a}$ ). The JCM interaction between the atom and the field is given by

$$
H_{\mathrm{JCM}}=g\left(|e\rangle\left\langle\left. g\right|_{a} a_{f}+a_{f}^{\dagger} \mid g\right\rangle\left\langle\left. e\right|_{a}\right),\right.
$$

where $a_{f}$ and $a_{f}^{\dagger}$ are the annihilation and creation operators of the field mode respectively. The joint density matrix for the atom-field system evolves with time as

$$
\rho_{a f}=\sum_{n=0}^{\infty} P_{n} \rho_{n}
$$

where

$$
\begin{aligned}
\rho_{n} & =\cos ^{2}\left(\frac{\Omega_{n} t}{2}\right)|e, n\rangle\left\langle e,\left.n\right|_{a f}\right. \\
& -i \cos \left(\frac{\Omega_{n} t}{2}\right) \sin \left(\frac{\Omega_{n} t}{2}\right)|e, n\rangle\left\langle g, n+\left.1\right|_{a f}\right. \\
& +i \cos \left(\frac{\Omega_{n} t}{2}\right) \sin \left(\frac{\Omega_{n} t}{2}\right)|g, n+1\rangle\left\langle e,\left.n\right|_{a f}\right. \\
& +\sin ^{2}\left(\frac{\Omega_{n} t}{2}\right)|g, n+1\rangle\left\langle g, n+\left.1\right|_{a f}\right.
\end{aligned}
$$

where $\Omega_{n}=2 g \sqrt{n+1}$ is the Rabi frequency.

We cannot exactly compute the total atom-field entanglement in the mixed state given by the above Eq.(4). 
However, we can obtain an estimate a lower bound on the entanglement using the projection to a $2 \times 2$ subspace discussed earlier. To see this, consider the field state being projected into the subspace spanned by $|n\rangle_{f}$ and $|n+1\rangle_{f}$. As this is a local action on the field, it cannot increase the entanglement. The resulting state is

$$
\rho_{a f}^{n}=\left(\begin{array}{cccc}
P_{n-1} S_{n-1}^{2} & 0 & 0 & 0 \\
0 & P_{n} S_{n}^{2} & P_{n} i C_{n} S_{n} & 0 \\
0 & P_{n} i C_{n} S_{n} & P_{n} C_{n}^{2} & 0 \\
0 & 0 & 0 & P_{n+1} C_{n+1}^{2}
\end{array}\right)
$$

where

$$
C_{n}=\cos \left(\frac{\Omega_{n} t}{2}\right), S_{n}=\sin \left(\frac{\Omega_{n} t}{2}\right) .
$$

Before proceeding to the evaluation of entanglement based on the above formula, we would check the separability of the above state. To prove the inseparability of this matrix we compute the eigenvalues of the partially transposed matrix. If one of the eigenvalues is negative then $\rho_{a f}^{n}$ is inseparable [22]. The existence of the negative eigenvalue reduces to the condition

$$
\left(P_{n} C_{n} S_{n}\right)^{2}>P_{n-1} P_{n+1}\left(C_{n+1} S_{n-1}\right)^{2} .
$$

Substituting $P_{n}, P_{n+1}$ and $P_{n-1}$ from Eq.(2) in the above expression we obtain the condition

$$
\Lambda_{n}=\left(C_{n} S_{n}\right)^{2}-\left(C_{n+1} S_{n-1}\right)^{2}>0,
$$

which is independent of $\langle n\rangle$ (i.e. of the temperature). We plot the expression $\Lambda_{n}$ with time $t$ in Fig.1 for three values of $n$.

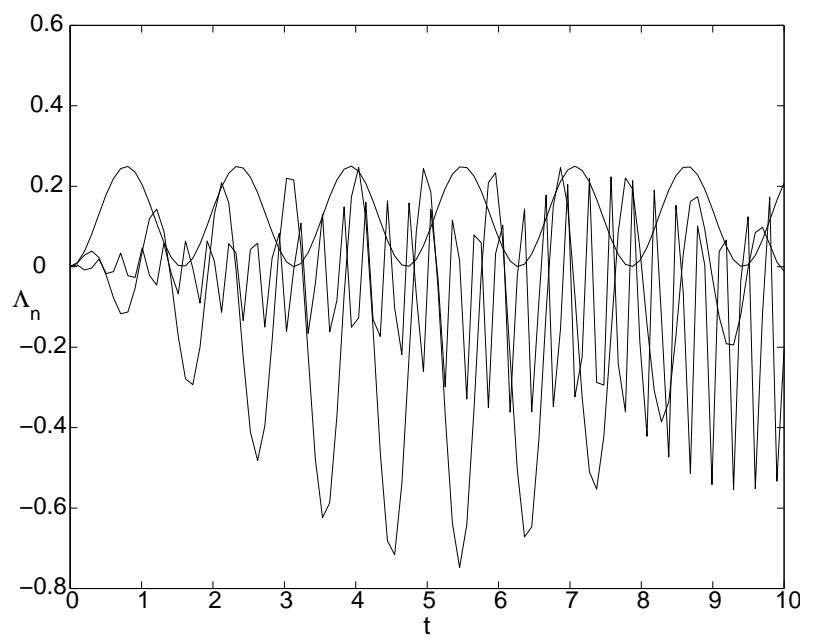

FIG. 1. Plots of the time variation of the inseparability expression $\Lambda$ for three values of $n$. The fastest oscillating curve is for $n=100$, the next fastest for $n=10$ and the slowest oscillating curve is for $n=0$.

From Fig.1, we see that the expression $\Lambda_{n}$ is positive for some intervals of time for each $n$ implying $\rho_{a f}^{n}$ is entangled during those intervals of time. The entire atomcavity state $\rho_{a f}$ is thus also entangled in those intervals of time (otherwise, no local projection would have given an entangled outcome). Entanglement thus arises due to JCM interaction of an atom and a field in a thermal state irrespective of the temperature of the field. Note that this is a rigorous result because even if $\Lambda$ is negative for just one specific value of $(n, t)$, then a local projection at that $t$ will result in the entangled outcome $\rho_{a f}^{n}$ with a finite probability. This would mean that prior to the local measurement, entanglement was present in the atom-field state at that time.

Based on the plots in Fig.1. we heuristically justify the following conjecture: Entanglement is present at all times except at $t=0$. The conjecture relies on the observed behaviour that for higher values of $n$, oscillations of $\Lambda_{n}$ (separability) with $t$ are faster. Consider an arbitrarily small interval of time $\delta t<\epsilon$. By going to sufficiently high $n$, one can always find that $\Lambda_{n}$ has a period smaller than $\delta t$ and thereby $\rho_{a f}^{n}$ is entangled for an interval of time within $\delta t$. We can choose the time interval $\delta t$ smaller and smaller, so that eventually there is entanglement at all instants of time.

We now briefly comment on the alternative scenario where the atom is in the initial thermal state

$$
\lambda|e\rangle\langle e|+(1-\lambda)| g\rangle\langle g|,
$$

where $\lambda /(1-\lambda)=\exp (-\Delta E / k T)(\Delta E$ being the energy difference between $|e\rangle$ and $|g\rangle$ and $T$ the temperature). The field is assumed to start in a pure Fock state $|n\rangle$. As the JCM is completely symmetric between the atomic and field operators, we can exchange the field and the atom states and have the same entanglement. This new scenario is equivalent to just two levels of the field involved in interaction with a pure atom. As shown earlier this also leads to entanglement at all temperatures of the atom.

The case when both the atom and the field are in thermal states is much more complex. In this case we expect that there is a cut-off temperature above which there is no entanglement. At infinite temperature, of course, all entanglement disappears as the total state is just proportional to the identity. However, our projection method can only lead to lower bounds as the failure of our method to produce entanglement by local projections does not imply that entanglement does not exist in the original mixed state. So, until there an operational necessary and sufficient condition for separability of $2 \times N$ density matrices, we cannot fully address this case. 


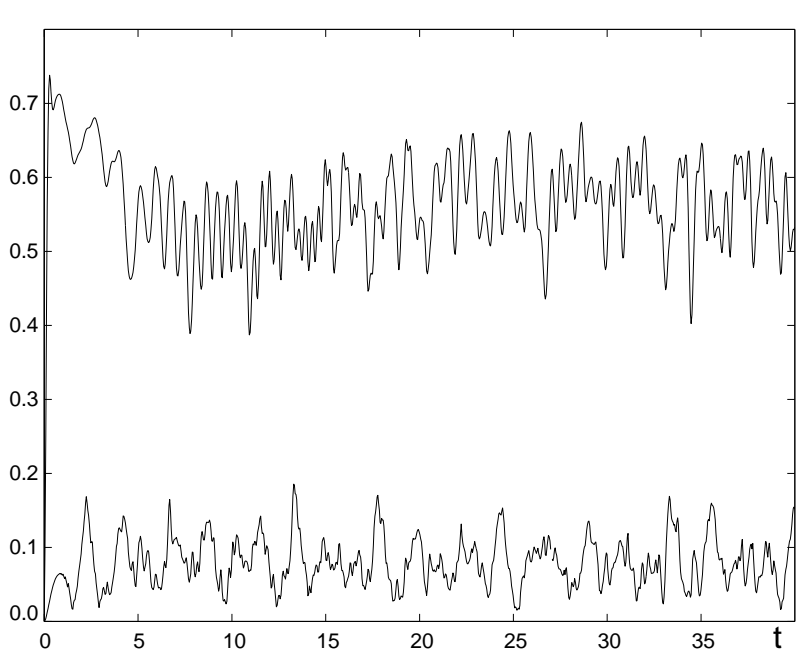

FIG. 2. Plots of the time variation of the mutual information and the entanglement in a thermal JCM model with $\langle n\rangle=10$. The upper curve represents mutual information and the lower curve represents entanglement.

As mentioned earlier, if we evaluate the entanglement of $\rho_{a f}^{n}$ and take an average over all possible values of $n$ (weighted by the probability of obtaining $\rho_{a f}^{n}$ ), we should get a lower bound on the entanglement of the state $\rho_{a f}$. This holds because $\rho_{a f}^{n}$ is the result of a local projection on $\rho_{a f}$. We have computed this lower bound on entanglement and the mutual information for initial thermal states with various mean photon numbers (e.g. a plot for the mean photon number $\langle n\rangle=10$ is shown in Fig. 2 ). We find, as we proved earlier, that JCM generates entanglement at any temperature of the cavity field, no matter how high. Moreover, though it starts from zero, it never completely vanishes. This supports our earlier conjecture that entanglement is present at all times except at $t=0$. Mutual information, on the other hand, quantifies total correlations and is therefore greater or equal to entanglement as seen in Fig. 2. Classical correlations are, of course, always seen to be present and this means that the total state is never of the product form at $t>0$ due to the JCM interaction. The mutual information is a good measure of classical correlations when entanglement is absent, since then total correlations are equal to classical correlations.

We have shown that entanglement can be generated from a very small amount of purity existing in an overall very mixed state. In principle, a $2 \times N$ system can be entangled even if the total entropy is as high as $\log N$ (the maximum being $1+\log N$ ). This is an important result among attempts to relate mixedness and entanglement [23]. This means in principle, one could entangle a microscopic system in a pure state with a macroscopic system in a thermal state, as suggested by the results in [24]. Our results should have implications for quantum computation with mixed states 25]. This kind of entanglement is probably involved in the functioning of Shor's algorithm with only one pure qubit 26]. Further studies could involve other natural interactions. For example, one could study how the creation of entanglement is affected by the off resonant interaction of the atom and the field. It is also an interesting and open question how efficiently one can entangle two partially mixed systems in general.

This research has been partly supported by the European Union, The UK Engineering and Physical Sciences Research Council and Hewlett-Packard. I. F.-G. would like to thank Consejo Nacional de Ciencia y Tecnologia (Mexico) Grant no. 115569/135963 for financial support.

[1] C. H. Bennett and D. P. DiVincenzo, Nature 404, 247 (2000); M.B. Plenio and V. Vedral, Cont. Phys. 39, 431 (1998); V. Vedral, The role of relative entropy in quantum information theory, quant-ph/0102094.

[2] M. A. Nielsen and I. L. Chuang, Quantum Computation and Quantum Information, Cambridge University Press, 2000.

[3] V. Vedral, M. B. Plenio, M. A. Rippin and P. L. Knight, Phys. Rev. Lett. 78, 2275 (1997); V. Vedral and M. B. Plenio, Phys. Rev. A 57, 1619 (1998).

[4] C. H. Bennett, D. P. DiVincenzo, J. A. Smolin and W. K. Wootters, Phys. Rev. A 54, 3824 (1996)

[5] W. K. Wootters, Phys. Rev. Lett 80, 2245 (1998).

[6] E. T. Jaynes and F. W. Cummings, Proc. IEEE 5189 (1963).

[7] B. W. Shore and P. L. Knight, J. Mod. Opt. 40, 1195 (1993).

[8] J. H. Eberly, N. B. Narozhny and J. J. SanchezMondragon, Phys. Rev. Lett. 20,1323 (1980)

[9] G. Rempe and H. Walther, Phys. Rev. Lett. 58353 (1987).

[10] M. Brune et. al., Phys. Rev. Lett. 76, 1800 (1996).

[11] S. Haroche and J. M. Raimond, Advan. Atom. Mol. Phys. 20, 347 (1985).

[12] S. M Barnett and P. L. Knight, Phys. Rev. A, 33, 2444 (1986).

[13] M. Tavis and F. W. Cummings, Phys Rev.,170, 279 (1968).

[14] A. M. Abdel-Hafez, A. S. F. Obada and M. M. A. Ahmad, Phys. Rev. A, 144, 530 (1987)

[15] J. Parker and C. R. Stroud Jr. , J. opt. Soc. Am. B 3, 96 (1986).

[16] P. L. Knight, Physica Scripta, 33(T12), 51 (1986).

[17] S.J. D. Phoenix and P. L. Knight, Ann Phys. 186, 381 (1988); S. J. D. Phoenix and P. L. Knight, Phys. Rev. A 44, 6023 (1991).

[18] A. Ekert and P. L. Knight, Amer. J. Phys., 63, 415 (1995).

[19] W. K. Wootters, Phys. Rev. Lett. 80 2245-2248 (1998).

[20] L. Henderson and V. Vedral, Phys. Rev. Lett. 84, 2263 (2000).

[21] R. S. Ingarden, A. Kossakowski and M. Ohya, " Infor- 
mation Dynamics and Open Systems - Classical and Quantum Approac", (Kluwer Academic Publishers, Dordrecht, 1997).

[22] M. Horodecki, P. Horodecki, R. Horodecki, Phys. Lett. A 223, 1 (1996).

[23] S. Bose and V. Vedral, Phys. Rev. A 61, 040101(R), (2000).

2
[24] S. Bose, K. Jacobs and P. L. Knight, Phys.Rev. A 59, 3204 (1999).

[25] S. Bose, L. Rallan and V. Vedral, Phys. Rev. Lett. 85, 5448 (2000).

[26] M. Mosca, PhD Thesis, University of Oxford (1999); S. Parker and M. B. Plenio, Phys. Rev. Lett. 85, 3049 (2000); S. Parker and M. B. Plenio, quant-ph/0102136. 